\title{
An Improved B-RRT* Method for UAV Path Planning
}

\author{
Shuai Zhao, Zhiyuan Shen ${ }^{+}$and Wenbin Wei \\ College of Civil Aviation, Nanjing University of Aeronautics and Astronautics, China
}

\begin{abstract}
With the fast development of science and technology, unmanned aerial vehicle (UAV) becomes more popular. As the use range of UAV expanding from the military to the civilian field, its path planning problem attracts lots of attentions. The conventional B-RRT* algorithm can not obtain the direct route for UAV flight. In this paper, an improved B-RRT* algorithm is proposed. The proposed algorithm is combined the dynamics constraints of the UAV with the node expansion of the extended tree. It firstly adopt to the backward fan-shaped area sampling method to improve the convergence speed from the selection strategy of random points. Then an adaptive step size is used to overcome the shortcomings of the limitations of growth at the adjacent of obstacles. Simulation results show that the proposed algorithm has a shorter path planning length with stronger stability, compared with other competing path planning method.
\end{abstract}

Keywords: path planning, dynamic constraint, backward fan-shaped area sampling, adaptive step size.

\section{Introduction}

With the progress of electronic information technology and the development of science and technology, UAV (Unmanned Aerial Vehicle) for its light, flexible, low cost and high reliability is becoming more and more popular. In the early 21 st century, the application of UAV has a broader prospect, and the use range of UAV has expanded from the military to the civilian field [1] .Trajectory planning is an important issue in UAV navigation and robotics.

Sample-based trajectory planning algorithms has proven to be effective in solving trajectory planning problems. Probability Roadmap Method (PRM) [2] and Rapidly-exploring Random-Tree (RRT) [3]-[5] is the current two main sampling methods. The RRT algorithm converges fast, and the route search of the tree is easier than the route search of the graph, which is widely used at present. However, there are still some shortcomings of the RRT algorithm, many domestic and foreign scholars have made lots of research. Many improved RRT algorithms have been proposed to meet different application scenarios. In order to improve node efficiency, Kuffner and Lavalle proposed RRT-connect in 2000 [6]. The following year, they proposed Bidirectional-RRT, which generates two trees in parallel starting respectively from the starting point and the target point until the two trees meet, to accelerate the convergence of the algorithm [7]. C. Urmson and R. Simmons proposed a heuristic biased algorithm that leads the tree to grow in the target area and improves the efficiency of the algorithm [8]. Karaman and Frazzoli first proposed the RRT* algorithm, which effectively improves the problem that is not the optimal solution generated by the basic RRT algorithm [9]. M. Jordan and A. Perez proposed the B-RRT* algorithm, using a slightly variant greedy RRT-connect as a heuristic function to connect two random trees [10]. Ahmed Hussain Qureshi and Yasar Ayaz proposed IB-RRT* algorithm, using a bidirectional tree approach, which allows the algorithm to the optimal path converge quickly through the heuristic function inserted by the smart sample [11].

In this paper, some improvements have been made on the basis of B-RRT* and combines the dynamics constraints of the UAV to make the generated trajectory be directly used by the UAV. Then, the method of

Corresponding author. Tel.: +86-25-84893461; fax: +86-25-84893461.

E-mail address: shenzy@nuaa.edu.cn 
sampling in the backward fan-shaped area is adopted to reduce the range selection of random points, so as to reduce the number of iterations and accelerate the convergence of the algorithm. Finally, we adjust adaptive step size in an open/obstacles environment to improve the speed of the algorithm.

\section{UAV Kinematic Model}

According to the space environment and threat parameters of the mission area, UAV is required to make a collision-free trajectory planning from the start point to the target point, and satisfy the flight performance constraints of the UAV. At the same time, the path length is short. Assuming the UAV is flying at a constant speed and altitude, the kinetic constraints can be described using the Dubins model:

$$
\begin{aligned}
& \dot{x}=v \cos \psi \\
& \dot{y}=v \sin \psi \\
& \dot{\psi}=\left(v / R_{\min }\right) u \\
& \varphi \leq \varphi_{\max } \\
& \text { Path } \leq M
\end{aligned}
$$

where $(x, y)$ is the position of the UAV in the inertial coordinate system. The mean of $v$ is the speed of the $\mathrm{UAV} . \psi$ is the heading angle. $R_{\min }$ is minimum turning radius and $u \in\{-1,0,1\} . \varphi_{\max }$ is maximum turning angle. $M$ is maximum track length.

\section{UAV Path Planning Algorithm Based on Improved B-RRT*}

Although the B-RRT* algorithm improves the convergence rate of the algorithm by utilizing the simultaneous bidirectional tree growth and preserves the probability integrity of the RRT* algorithm, the B-RRT* algorithm still needs to be improved in some places. The path optimization is achieved by improving the sampling strategy in the backward fan-shaped area and adjusting the adaptive step size.

\subsection{Backward Fan-shaped Area Sampling}

When the UAV is in normal flight, the maximum turning angle must be satisfied in order to make the track fly [12]. As shown in the Fig. 1, when the random point ${ }^{x_{\text {rand }}}$ is within the feasible region, the node expansion does not change. When not in the feasible region, at this time the introduction of random variables $\xi \in[-1,1]$, through the heading $\xi \times \varphi_{\max }$ to determine the choice of random points, generate the insertion point is as follows:

$$
x_{\text {new }}= \begin{cases}x_{\text {near }}+\mu \times \frac{x_{\text {rand }}-x_{\text {near }}}{\left|x_{\text {rand }}-x_{\text {near }}\right|} \quad & x_{\text {rand }} \in Q \\ x_{\text {near }}+\mu \times\left[\sin \left(\xi \times \varphi_{\max }\right),\right. & \left.\cos \left(\xi \times \varphi_{\text {max }}\right)\right] \quad x_{\text {rand }} \notin Q\end{cases}
$$

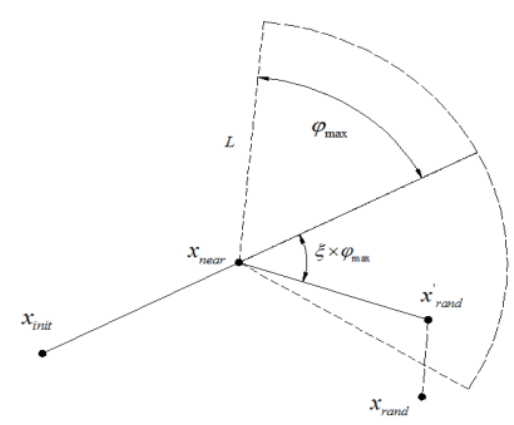

Fig. 1: Improved random point selection.

\subsection{Adaptive Step Size}

Aiming at the problem that the fixed step grows in the vicinity of the obstacle, IB-RRT* algorithm introduces the concept of adaptive step length. Through NearObstacle $\left(x_{1}, 2 \mu_{\max }\right)$, find and calculate the distance $D_{i}$ of the nearest obstacle and calculated according to the step length: 


$$
\mu=\frac{D_{i}}{K}\left(\mu_{\min } \leq \mu \leq \mu_{\max }\right)
$$

According to the equation. The closer the obstacle, the closer the step is $\mu_{\min }$. When there is no obstacle in fan-shaped area, the step size is $\mu_{\max }$.

The specific steps of the UAV trajectory planning IB-RRT* algorithm method are as follows:

Step1: Initializes the search path to include only the starting point $x_{\text {init }}$ and target point $x_{\text {goal }}$;

Step2: If $\left|x_{\text {init }}-x_{\text {goal }}\right| \leq L$, the search path return Path $=\left\{\left(x_{\text {init }}, x_{\text {goal }}\right)\right\}$, otherwise return to Step4;

Step3 : If $T_{1}$ and $T_{2}$ meets, return the Path $=\left\{\left(T_{1}, T_{2}\right)\right\}$;

Step4 : For the search tree $T_{1}$, call the extension node function. Sample points $x_{\text {rand }}$ are generated in the backward fan-shaped area, and find the nearest tree node $x_{\text {near }}$ in the extended tree node $x_{\text {rand }}$. Generate insertion points $x_{\text {new }}$ in the middle. Judge whether the total distance of the path generated is smaller than before. If so, add it to the search tree $T_{1}$;

Step5 : In the process of extending the node, if the insertion point $x_{n e w}$ encounters an obstacle, call dynamic adaptive step function to adjust step length. The random node generation function to generate a random node. Add this node to the search tree $T_{1}$;

Step6: For the search tree $T_{2}$, its expansion process is same as $T_{1}$;

Step7: In each iteration, when one tree expands, it attempts to connect the nearest node of another tree to extend the new node, then exchange order, continue circulation. Set the maximum number of iterations $\mathrm{M}$, if can not find the target point in $\mathrm{M}$, the algorithm fails to return, otherwise return to Step3.

\section{Simulation and Results}

\subsection{Parameter Setting}

In order to verify the validity of the proposed algorithm, using Matlab tool programming to build a simulation environment. Simulation experiments were performed on Intel (R) Xeon (R) $2.4 \mathrm{GHz}, 2 \mathrm{~GB}$ memory computer. Suppose the UAV flight area $1000 \mathrm{~m} \times 1000 \mathrm{~m}$, the starting point coordinates $(100,100)$, the target point coordinates $(900,900)$.In the flight area, randomly generate 100 circular area obstacles with different radius. Probability $=0.5, \varphi_{\max }=60^{\circ}$, the adaptive step size is between $(10,20)$.

\subsection{Simulation And Results}

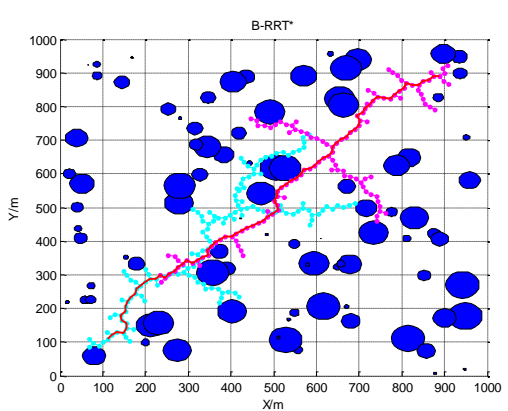

(a) B-RRT* expansion distribution.

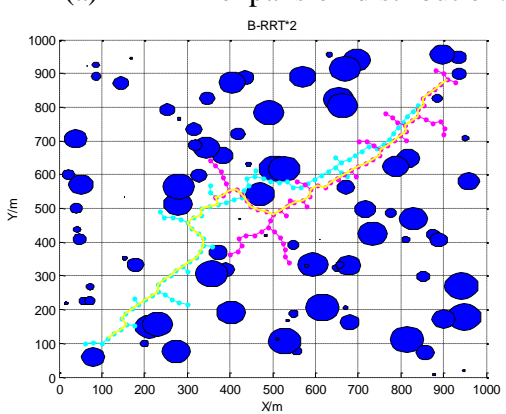

(c) B-RRT*2 expansion distribution

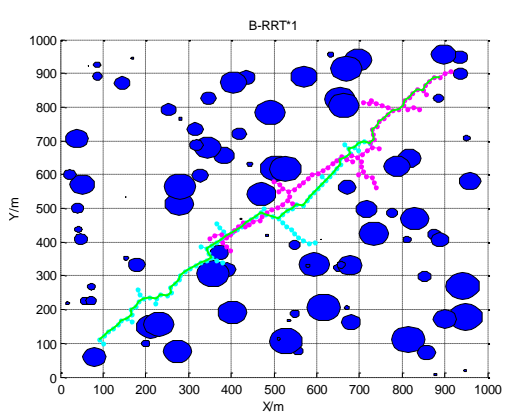

(b) B-RRT*1 expansion distribution.

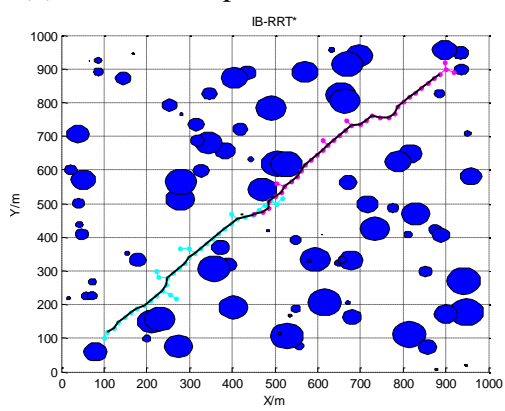

(d) IB-RRT* expansion distribution.

Fig. 2: Expansion tree distribution with different path planning algorithms. 
As can be seen from the figure above, B-RRT*1(Fig. 2b) uses backward fan-shaped area sampling. It can be seen that exploration point to reduce and the direction of tree generation is more definite than B-RRT*(Fig.2 a).B-RRT*2 (Fig. 2c) is adaptive step size that improves the limitations of growing near obstacles. IB-RRT* (Fig. 2d) combining the methods of the two mentioned earlier, the generation graph is better.

Comparing the four algorithms involved in this article, get the UAV planning path as follows:

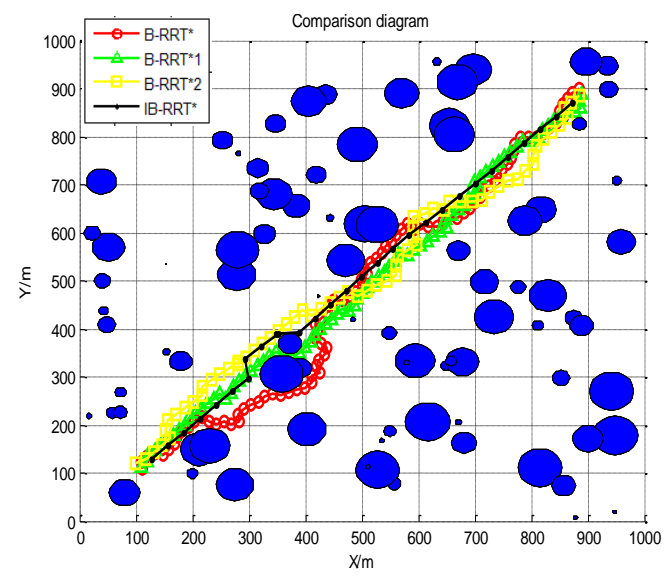

Fig. 3: Final planned path with competing algorithms.

\subsection{Stability Analysis}

Because of the randomness of the algorithm, simulate 100 times to verify whether the result is stable. The simulation results of 100 times are compared as shown in the following Table. 1. The IB-RRT* algorithm performs well in three indicators, indicating that it has stability.

Table 1: Simulation 100 times

\begin{tabular}{lccc}
\hline & Number of points(n) & The length of the path $(\mathrm{m})$ & Consume time $(\mathrm{s})$ \\
\hline B-RRT $*$ & 101 & $1.3413 \mathrm{e} 03$ & 0.3056 \\
B-RRT $* 1$ & 82 & $1.2362 \mathrm{e} 03$ & 0.1621 \\
B-RRT $* 2$ & 89 & $1.2791 \mathrm{e} 03$ & 0.2023 \\
IB-RRT & 68 & $1.1629 \mathrm{e} 03$ & 0.1627 \\
\hline
\end{tabular}

\subsection{Asymptotically Optimal Analysis}

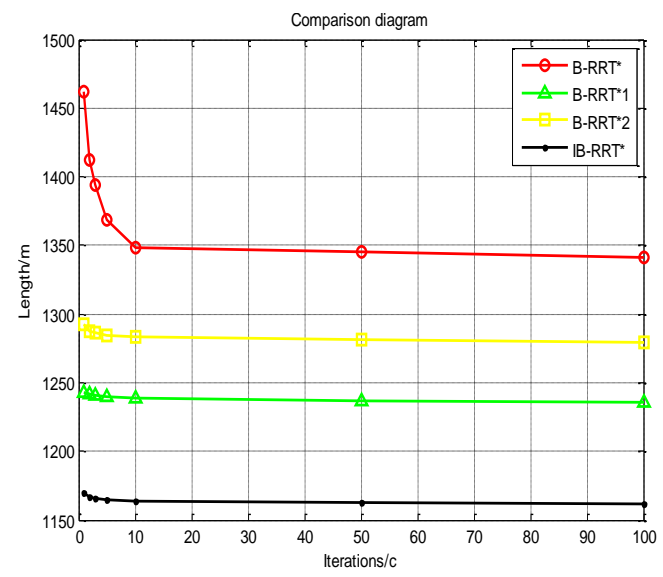

Fig. 4: Asymptotically optimal comparison.

The asymptotic behavior of the four algorithms is shown in the Fig. 4. B-RRT* behaves as usual in the previous cases, but with the number of simulations increasing, it can be clearly seen that it has asymptotic optimality. The other three algorithms are better too and it can be seen from the Fig. 4 that the optimal size relationships of the four algorithms is IB-RRT*> B-RRT*1>B-RRT*2> B-RRT*. 
The above results of computer simulation show that the improved B-RRT* algorithm is superior to the original B-RRT* and can generate the direct path for UAV flight. The effectiveness and the superiority of the proposed algorithm are verified.

\section{Conclusion}

Based on the excellent features of heuristic information, probability completeness and asymptotic optimality in the original B-RRT*, this paper proposes an improved IB-RRT* algorithm. The proposed algorithm combines the UAV dynamics constraints into the expansion of the tree nodes. The selection of insertion point by the backward fan-shaped area sampling, and adjusts adaptive step size to overcome the shortcomings of the limitation of growth at the adjacent of obstacles. The above simulation results show that, IB-RRT* algorithm performs best in four different expansion tree distribution, and the verification analysis shows, the trajectory stability, high repeatability and is closed to the optimal trajectory. The IB-RRT* algorithm to further improve the practicality and stability, can be better used in the UAV path planning.

\section{Acknowledgements}

The authors would like to thank the financial grant by National Natural Science Foundation of China (No.61501225)and China Postdoctoral Science Foundation (No.2016M601806).

\section{References}

[1] MIAO Yan-qing, JIN Lei TAN Wei-rong. A brief discussion on the development trend of airworthiness of civilian uav in China[J]. Research on airworthiness technology. 2014:29-32. (in Chinese)

[2] LAVAlLE S M, KUFFNER J J. Randomized Kino dynamic Planning [J].IEEE International Conference on Robotics \&Automation, 1999, 1(5):473-479.

[3] MELCHIOR N A,SIMMONS R. Particle RRT for Path Planning with Uncertainty[C]//IEEE International Conference on Robotics\&Automation.2007:1617-1624.

[4] Shi K, DennyJ, Amato NM, Spark PRM:Using RRTs within PRMs to efficiently explore narrow passages[C]//IEEE International Conference on Robotics and Automation, ICRA2014.Piscataway: IEEE, 2014, 4659-4666.

[5] Denny J, Marales M, Rodriguez S, et al. Adapting RRT growth for heterogeneous environments[C]// IEEE International Conference on Intelligent Robotics and Systems (IROS). Piscataway: IEEE, 2013, 1-7.

[6] Kuffner J, RRT-Connect S L V.An Efficient Approach to Single-query Path Planning IEEE International Conference on Robotics and Automation [J].San Francisco, 2000, 2:473-479

[7] LAVAlle S M, KUFFNER J. Randomized Kinodynamic Planning [J].The International Journal of Robotics Research, 2001, 20(5):378-400.DOI:10.1177/02783640122067453.

[8] Urmson C, Simmons R G.Approaches for Heuristically Biasing RRT Growth[C]//IROS,2003,2:1178-1183.

[9] Karaman S, Frazzoli E. Incremental Sampling-based Algorithms for Optimal Motion Planning[J].Robotics Science and Systems VI, 2010, 104.

[10] Jordan M, Perez A. Optimal Bidirectional Rapidly-exploring Random Trees, MIT-CSAIL-TR-2013-021[R]. Cambridge, UK: Computer Science and Artificial Intelligence Laboratory, 2013

[11] Qureshi A H, Ayaz Y. Intelligent Bidirectional Rapidly-exploring Random Trees for Optimal Motion Planning in Complex Cluttered Environments [J], Robotics and Autonomous Systems, 2015, 68:1-11.

[12] PENG Hui, WANG Lin, SHEN Lin-cheng. The modified RRT-based real-time route planning for UAV area target searching[J].Journal of National University of Defense Technology,2009,31(5):86-91.(in Chinese) 